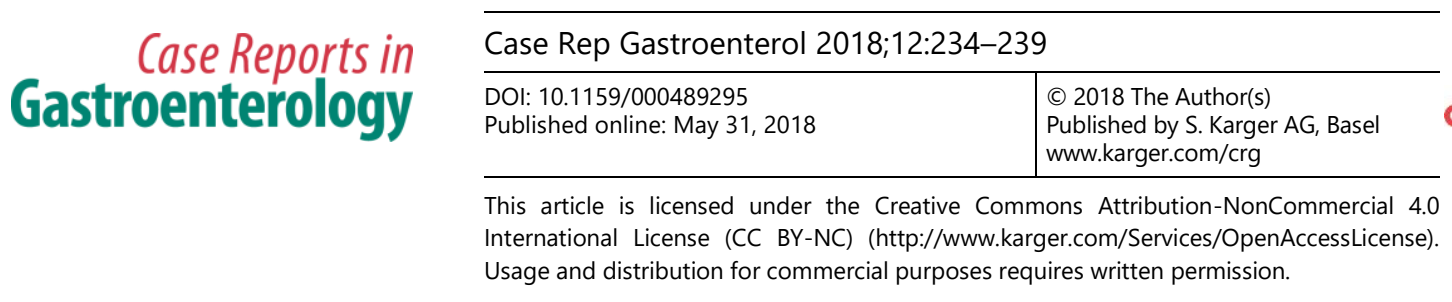

\title{
Esophageal Large-Cell Neuroendocrine Carcinoma with Inconsistent Response to Treatment in the Primary and Metastatic Lesions
}

\author{
Takashi Tomiyama $^{a}$ Masahiro Orino ${ }^{a}$ Koh Nakamaru $^{a}$

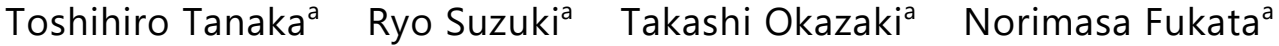 \\ Yugo Ando ${ }^{a}$ Naoyuki Danbara ${ }^{a}$ Toshiro Fukui $^{a}$ Chika Miyasaka $^{b}$ \\ Kazuichi Okazaki ${ }^{\mathrm{a}}$ \\ aDivision of Gastroenterology and Hepatology, Third Department of Internal Medicine, \\ Kansai Medical University, Osaka, Japan; ${ }^{b}$ Department of Pathology and Laboratory \\ Medicine, Kansai Medical University, Osaka, Japan
}

\section{Keywords}

Esophageal large-cell neuroendocrine carcinoma · Chemotherapy · Esophageal stenosis ·

Chemoradiotherapy

\begin{abstract}
Esophageal large-cell neuroendocrine carcinoma (NEC) is a rare malignant tumor that is characterized by high-grade malignancy and a poor prognosis. However, the rarity of esophageal NEC has prevented the development of an established treatment, and no reports have described a discrepancy in the effectiveness of cisplatin plus irinotecan between primary and metastatic lesions. A 43-year-old Japanese man was referred to our hospital with refractory epigastralgia. A previous gastrointestinal endoscopy had revealed a 50-mm type 2 tumor in the abdominal esophagus. The pathological findings indicated poorly differentiated squamous cell carcinoma. Contrast-enhanced computed tomography revealed a metastatic liver tumor.
\end{abstract}




\section{Case Reports in Gastroenterology}

Case Rep Gastroenterol 2018;12:234-239

DOI: 10.1159/000489295

(c) 2018 The Author(s). Published by S. Karger AG, Basel www.karger.com/crg

Tomiyama et al.: Esophageal Large-Cell NEC with Inconsistent Response to Treatment

One cycle of fluorouracil and cisplatin was not effective, and endoscopy was repeatedly performed. The pathological findings indicated a large-cell malignant tumor with tumor cells that were positive for CD56, synaptophysin, and $\mathrm{Ki}-67$ (>80\%). Based on a diagnosis of esophageal large-cell NEC with a metastatic liver tumor, the patient received cisplatin plus irinotecan biweekly. After 4 months, computed tomography revealed marked shrinkage of the metastatic tumor, but the patient complained of dysphagia. Endoscopy revealed enlargement of the primary tumor, which was then treated using radiotherapy plus fluorouracil and cisplatin. The primary tumor subsequently shrank, and the patient's symptoms were relieved, but the metastatic tumor grew. Thus, chemoradiotherapy could be an option for managing a primary esophageal large-cell NEC that does not respond to chemotherapy alone. However, the possibility of an inconsistent response to therapy in primary and metastatic lesions should be considered.

\section{Background}

Esophageal neuroendocrine carcinoma (NEC) is a rare malignant neoplasm that is characterized by a high malignant potential, rapid growth, and a poor prognosis [1]. However, the rarity of NEC has impeded the development of a standard chemotherapy regimen. Based on the clinical and histopathological similarity between NEC and small cell lung cancer (SCLC), the chemotherapeutic regimens for gastrointestinal NEC are generally the same as those for extensive SCLC, with one widely used option being etoposide (VP-16) plus cisplatin (CDDP) [2-5]. Nevertheless, evidence from the Japan Clinical Oncology Group study (JCOG9511) revealed that administering irinotecan (CPT-11) plus CDDP provides a better prognosis among patients with SCLC than does VP-16 plus CDDP [6]. Considering the similarity between NEC and SCLC, Japanese patients with metastatic esophageal NEC are commonly treated using CPT-11 plus CDDP $[7,8]$.

We report our experience with a case of esophageal large-cell NEC that had a unique chemotherapeutic response, as the metastatic and primary tumors responded differently to CPT-11 plus CDDP. The primary tumor responded to later concurrent radiotherapy and chemotherapy using 5-fluorouracil (5-FU) plus CDDP, although the metastatic tumor subsequently grew.

\section{Case Report}

A 43-year-old Japanese man had had a 2-month history of epigastralgia and abdominal fullness and had been diagnosed with stage IV esophageal cancer before being referred to our center for chemotherapy. A previous gastrointestinal endoscopy had revealed a 50-mm type 2 tumor in the abdominal esophagus, located $45 \mathrm{~cm}$ from the incisors. The pathological findings from the biopsy specimens revealed poorly differentiated squamous cell carcinoma. Contrast-enhanced computed tomography (CT) also revealed a 90-mm metastatic liver tumor in the right lobe. The patient did not have a history of esophageal cancer or any related risk factors (e.g., alcohol consumption or smoking) and was not receiving any medication.

A physical examination revealed severe hepatomegaly with tenderness. Laboratory findings revealed a white blood cell count of 5,000/ $\mu \mathrm{L}$ and a C-reactive protein level of $8.0 \mathrm{mg} / \mathrm{dL}$, but the level of squamous cell carcinoma antigen was not elevated. An initial cycle of 5-FU plus CDDP did not improve his symptoms or the laboratory findings. Repeated endoscopy revealed 


\section{Case Reports in Gastroenterology}

Case Rep Gastroenterol 2018;12:234-239

DOI: 10.1159/000489295

(c) 2018 The Author(s). Published by S. Karger AG, Basel www.karger.com/crg

Tomiyama et al.: Esophageal Large-Cell NEC with Inconsistent Response to Treatment

that the type 2 tumor at the abdominal esophagus had not changed (Fig. 1a). Hematoxylin and eosin staining of the second biopsy specimen revealed invasive large cells with significant cytoplasm, prominent nuclei, and vesicular chromatin (Fig. 1b). Immunohistochemical staining revealed that the tumor cells were positive for CD56 and synaptophysin (Fig. 1c, d), and that $>80 \%$ of the tumor cells were positive for Ki-67 (Fig. 1e). Contrast-enhanced abdominal CT revealed that the metastatic liver tumor had grown to a diameter of $120 \mathrm{~mm}$ (Fig. 1f). The serum neuron-specific enolase level was $109.5 \mathrm{ng} / \mathrm{mL}$ (normal range: $0-15$ ). Thus, the final diagnosis was metastatic large-cell NEC of the esophagus (grade 3).

The patient subsequently received chemotherapy using intravenous infusions of CTP-11 $\left(60 \mathrm{mg} / \mathrm{m}^{2}\right)$ plus CDDP $\left(30 \mathrm{mg} / \mathrm{m}^{2}\right)$ every other week. After 9 cycles over 4 months, his epigastralgia and abdominal fullness were relieved. CT revealed a marked reduction in the size of the metastatic liver tumor (from 120 to $75 \mathrm{~mm}$ in diameter; Fig. 2a). No severe adverse effects were observed during this round of treatment, although the patient complained of newly developed dysphagia, and repeat endoscopy revealed clear enlargement of the primary tumor (Fig. 2b). Thus, the patient received chemotherapy using concurrent CDDP and 5-FU (CDDP: $70 \mathrm{mg} / \mathrm{m}^{2}$ on days 1 and 29; 5-FU: $700 \mathrm{mg} / \mathrm{m}^{2}$ in continuous infusions on days 1-5 and 29-33) along with radiotherapy (60 Gy in 2-Gy doses, 5 days per week). Repeat endoscopy 1 month after completing this course of chemoradiotherapy revealed a dramatically smaller esophageal tumor (Fig. 3a), but an enlarged metastatic tumor (Fig. 3b).

The patient subsequently received biweekly treatment with CPT-11 plus CDDP to reduce the size of the liver tumor, but the additional 6 cycles of this treatment led to a chemotherapyrefractory status, with no response observed in the metastatic liver tumor. In addition, the metastatic tumor did not respond to subsequent weekly paclitaxel treatment. The patient ultimately died of liver failure 11 months after starting treatment.

\section{Discussion}

NEC is pathologically classified into small-cell and large-cell types. Primary esophageal large-cell NEC is very rare [2, 9], with no established treatment for it. However, with the similarity in the clinical course and histopathological features of NEC and SCLC, inoperable NEC is generally treated using the same chemotherapy regimen as for SCLC, regardless of whether it is of the small-cell or the large-cell type. Even in operable cases, adjuvant chemotherapy is part of the treatment, as it may provide a better prognosis than surgery alone $[1,10]$. CDDP is a critical cytotoxic agent and is usually combined with VP-16 or CPT-11 as the first line of treatment. Thus far, second-line and later treatments for esophageal NEC have remained poorly defined. Radiation therapy is another therapeutic option, although experience with this approach is limited [11].

The present case involved a unique clinical course, as the response of the metastatic tumor to treatment with CDDP plus CPT-11 was the opposite of the response observed in the primary tumor; the primary tumor responded to radiation therapy plus 5-FU and CDDP. This clinical course suggests that radiation therapy plus 5-FU and CDDP was able to control the primary esophageal tumor but not the metastatic liver tumor, making it difficult to reach an optimal solution to control both primary and metastatic lesions. Dysphagia due to growth of the primary tumor reduces the quality of life extremely, specifically in the later stages of the disease. Esophageal stenting is a palliative option for dysphagia. Although recent esophageal stents possess an antireflux technology, stenting of distal esophageal stenosis increases the risk of gastroesophageal reflux and aspiration, as the stent should pass the gastroesophageal 
junction [12]. In our patient, we opted for chemoradiotherapy to alleviate the dysphagia, resolving the issue in the patient. Nonetheless, the chemotherapeutic regimen of 5-FU plus CDDP and radiotherapy failed to control the growth of the metastatic lesion, indicating that though this combination might have been a good choice for controlling the primary lesion, it was not the optimal regimen to stop the growth of the metastatic lesion. Thus, although the combination of radiotherapy and CPT-11 increases the risk of radiation pneumonitis [13], concurrent radiotherapy and chemotherapy with CDDP and CPT- 11 may be a better choice in managing both primary and metastatic esophageal NEC.

\section{Statement of Ethics}

Informed consent was not obtained because the patient cannot be identified based on the presented data.

\section{Disclosure Statement}

None of the authors have any conflict of interest to disclose.

\section{References}

1 Ma Z, Cai H, Cui Y. Progress in the treatment of esophageal neuroendocrine carcinoma. Tumour Biol. 2017 Jun;39(6):1010428317711313.

2 Kuriry H, Swied AM. Large-Cell Neuroendocrine Carcinoma of the Esophagus: A Case from Saudi Arabia. Case Rep Gastroenterol. 2015 Oct;9(3):327-34.

3 Bongiovanni A, Riva N, Ricci M, Liverani C, La Manna F, De Vita A et al. First-line chemotherapy in patients with metastatic gastroenteropancreatic neuroendocrine carcinoma. Onco Targets Ther. 2015 Dec;8:3613-9.

4 Heetfeld M, Chougnet CN, Olsen IH, Rinke A, Borbath I, Crespo G et al.; Other Knowledge Network Members. Characteristics and treatment of patients with G3 gastroenteropancreatic neuroendocrine neoplasms. Endocr Relat Cancer. 2015 Aug;22(4):657-64.

5 Lee SY, Choi YJ, Chang WJ, Shin SW, Kim YH, Kim ST. The role of chemotherapy and/or octreotide in patients with metastatic gastroenteropancreatic and hepatobiliary neuroendocrine carcinoma. J Gastrointest Oncol. 2014 Dec;5(6):457-62.

6 Noda K, Nishiwaki Y, Kawahara M, Negoro S, Sugiura T, Yokoyama A et al.; Japan Clinical Oncology Group. Irinotecan plus cisplatin compared with etoposide plus cisplatin for extensive small-cell lung cancer. N Engl J Med. 2002 Jan;346(2):85-91.

7 Okuma HS, Iwasa S, Shoji H, Takashima A, Okita N, Honma Y et al. Irinotecan plus cisplatin in patients with extensive-disease poorly differentiated neuroendocrine carcinoma of the esophagus. Anticancer Res. 2014 Sep;34(9):5037-41.

8 Funakoshi S, Hashiguchi A, Teramoto K, Miyata N, Kurita S, Adachi M et al. Second-line chemotherapy for refractory small cell neuroendocrine carcinoma of the esophagus that relapsed after complete remission with irinotecan plus cisplatin therapy: case report and review of the literature. Oncol Lett. 2013 Jan;5(1):117-22.

9 Wilson CI, Summerall J, Willis I, Lubin J, Inchausti BC. Esophageal collision tumor (large cell neuroendocrine carcinoma and papillary carcinoma) arising in a Barrett esophagus. Arch Pathol Lab Med. 2000 Mar;124(3):411-5.

10 Deng HY, Ni PZ, Wang YC, Wang WP, Chen LQ. Neuroendocrine carcinoma of the esophagus: clinical characteristics and prognostic evaluation of 49 cases with surgical resection. J Thorac Dis. 2016 Jun;8(6):1250-6.

11 Tustumi F, Takeda FR, Uema RH, Pereira GL, Sallum RA, Cecconello I. Primary neuroendocrine neoplasm of the esophagus - report of 14 cases from a single institute and review of the literature. Arq Gastroenterol. 2017 Jan-Mar;54(1):4-10.

12 Hindy P, Hong J, Lam-Tsai Y, Gress F. A comprehensive review of esophageal stents. Gastroenterol Hepatol (N Y). 2012 Aug;8(8):526-34. 
Tomiyama et al.: Esophageal Large-Cell NEC with Inconsistent Response to Treatment

13 Yamada M, Kudoh S, Hirata K, Nakajima T, Yoshikawa J. Risk factors of pneumonitis following chemoradiotherapy for lung cancer. Eur J Cancer. 1998 Jan;34(1):71-5.
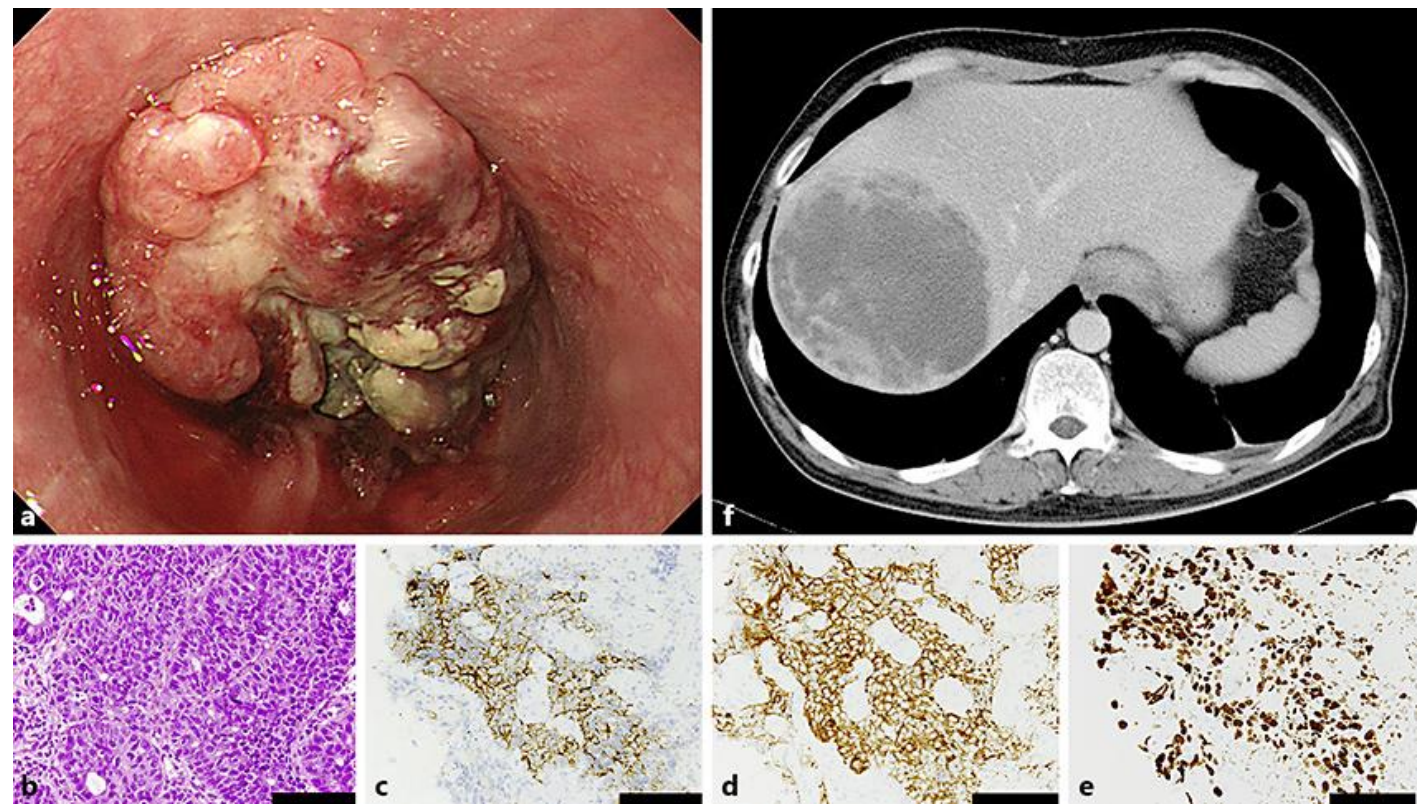

Fig. 1. Clinical findings prior to cisplatin plus irinotecan treatment after 1 cycle of fluorouracil plus cisplatin chemotherapy. a The endoscopic findings revealed a 50-mm semicircular invasive ulcerative tumor in the abdominal esophagus. b Hematoxylin and eosin staining of the biopsy specimen revealed a large-cell neoplasm. Immunohistochemistry testing for CD56 (c), synaptophysin (d), and Ki-67 (e) revealed that the tumor was a neuroendocrine carcinoma (grade 3). We certified antibody accuracy using tissue microarray specimens, and compared the staining magnitude between our specimens and the known TMA samples. Scale bars, $100 \mu \mathrm{m}$. f Contrast-enhanced computed tomography revealed a metastatic tumor with a diameter of $120 \mathrm{~mm}$ in the right lobe of the liver which enlarged after 1 cycle of fluorouracil plus cisplatin chemotherapy. 

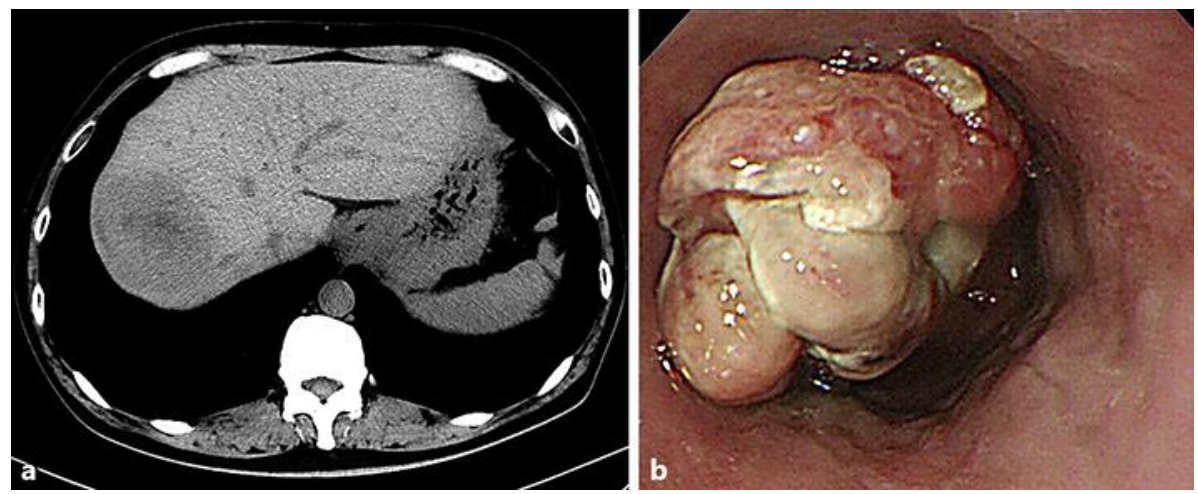

Fig. 2. Findings after 9 cycles of cisplatin plus irinotecan chemotherapy. a Computed tomography revealed marked shrinkage of the metastatic liver tumor (from 120 to $75 \mathrm{~mm}$ in diameter). b Repeat endoscopy revealed an enlarged primary tumor.
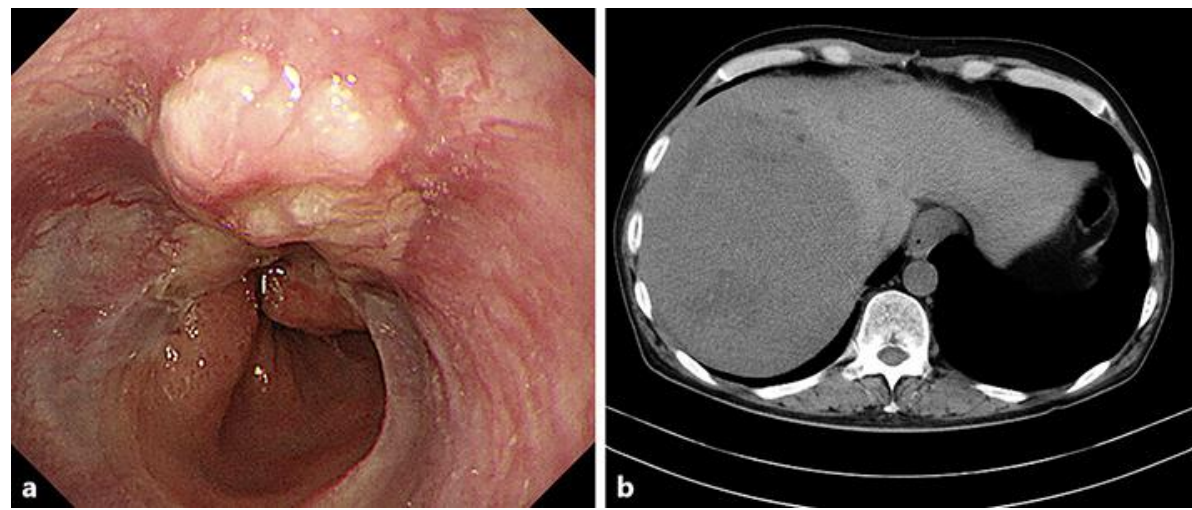

Fig. 3. Findings after radiotherapy plus fluorouracil and cisplatin chemotherapy. a Repeat endoscopy revealed marked shrinkage of the primary tumor. $\mathbf{b}$ Computed tomography revealed an enlarged metastatic tumor (from $75 \mathrm{~mm}$ after the first series of chemotherapy to $120 \mathrm{~mm}$ in diameter). 